\title{
MODELLING OF NONLINEAR DAMPERS UNDER LOW-AMPLITUDE VIBRATION
}

\author{
SG Demiryurek \\ The University of Sheffield, Sheffield, United Kingdom \\ A Krynkin \\ The University of Sheffield, Sheffield, United Kingdom \\ J Rongong \\ The University of Sheffield, Sheffield, United Kingdom
}

\section{ABSTRACT}

Particle dampers can suppress structural vibration over a broad range of frequencies, which makes them attractive in comparison to many other passive damping technologies. They constitute a cavity filled with particles. Energy dissipation from particle dampers depends on many parameters including, size of the cavity, diameter of the particles, shape of the damper, filling ratio, material properties of the particles and the volumetric ratio between the cavity and particles. Performance changes with the amplitude of the excitation and, to a lesser extent, the frequency. This study focuses on the energy dissipation in the granular material that fills particle dampers as the damper is subjected to low-amplitude dynamic load. The behaviour of this material is modelled using the Discrete Element Method (DEM) for a specific case: a tube-shaped cavity filled with spherical particles. An equivalent continuum model is proposed for the granular material and the Finite Element Method (FEM) is used to simulate the response of a damper subject to structural vibration. This study shows how the equivalent material model can be used to predict amplitude dependent behaviour in particle dampers under lowamplitude excitation.

\section{INTRODUCTION}

Controlling vibration and damping mechanisms are major area of interest within the field of structural vibration especially in engineering and machining process. Types of vibration controlling mechanisms are divided into two main arms: active and damping. Passive damping treatments have received particular attention since they do not require additional energy ${ }^{1}$. There are numerous methods for the passive damping treatments like viscoelastic dampers, tuned mass dampers, friction dampers, etc. These dampers do not require additional energy to reduce the structural vibration which makes these applications as low-cost damping treatments. However, there are limitations of passive dampers such as leakage issues, temperature intolerance, etc. While there are uncertainties on the usage of these passive damping methods, there is an alternative damper known as particle dampers. Due to the simplicity and efficiency of the particle dampers, there is an increasing interest on them. Particle damper is made of a cavity filled with small sized particles which could be made of metals, polymers, ceramics ${ }^{2}$. These dampers can be a part of the main structure or can be attached to the main structure. While there is external excitation on the system, kinetic energy is absorbed by the particle damper through the inelastic collisions provided by the contacts between particle-particle interactions and particle-cavity wall interactions ${ }^{3}$. In addition to this, the excitation level changes the position of the particles in the damper which, in total, shapes the damping level. When the system at resting position, the gravity affect the particles and they have static forces, which is mentioned as hydrostatic pressure ${ }^{4}$. Low amplitude of dynamic load 


\section{Proceedings of the Institute of Acoustics}

keeps these granular structures in their stable positions where a small amount of energy is reduced from the main structure. Under the dynamic load, the particles have dynamic and friction forces acting on them to move or vibrate. They change their positions and contact surfaces as a result of the load. While these changes are getting increased, the contact forces are changed, as well. Linked to the excitation level, the contact forces can lock the particles or can cause sliding between them. The amplitude exceeding the gravitational forces and friction forces between the particle contact forces, these particles start moving around in the cavity. The amplitude dependency term is defined as these explained behavioural changes in the particles state which changes the damping effectivity ${ }^{5}$.

Particle dampers are affected by the other system parameters, as well: Size, amount and shape of the particles are in these parameters, since the contact mechanism changes with all these inclusions of the system ${ }^{6}$. Several attempts have been made to reveal the effect of parameters of particle dampers using analytical, numerical and experimental methods. Analytical methods are not inclusive in terms of reflecting the physical arrangements and contact properties; however, numerical attempts are produced using these analytical assumptions and they are further developed to show the parameters of the particle dampers. Discrete Element Method (DEM) is used in general as method of numerical studies of the particle dampers. Experimental studies have a great impact in order to finalise the real application of analytical and numerical approaches of the particle dampers ${ }^{7}$. However, experimenting vast variety of parameters is quite expensive. Therefore, a systematic understanding on particle dampers have been tried using numerical methods, mostly.

In this paper a novel modelling technique is developed to express the properties of particle dampers as properties of an effective medium. Equivalent material modelling of granular state is required and developed using the same-sized spherical particles in tube-shaped cavity. This is used to solve structural vibration problem with granular material included in form of the effective medium. The FEM model will then be available to be used to obtain loss factor associated with the equivalent material properties.

\section{METHODOLOGY}

\subsection{DEM Model}

In order to study the packed granular structures, contact models have been developed using the analytical relationship between the neighbouring particles in a pack. DEM is general and widely used approach of modelling the stress-strain and force-displacement relationships between the granules. The iterative process is used to identify stress definitions of individual bodies in a pack under gravitational force where the displacement was assumed to be zero. This stress values are used to define the force-displacement relations under dynamic load. The iterative process continues until there is a convergence in the solution ${ }^{8}$.

These series of calculations were used in order to attain the equivalent properties of the granular state. The vector traction force was defined for three spatial dimensions where Lamế moduli $(\mu, \lambda)$ (Eq. 1) were inserted into the equations at which the displacement was assumed to have a value of bigger than zero in the resting state of two-neighbouring granules (Figure 1). From this point, the force which changes the state of the particles 


\section{Proceedings of the Institute of Acoustics}

with uniform infinitesimal displacement is applied. By following these calculations, average stress and average strains on a certain particle were defined ${ }^{9}$.

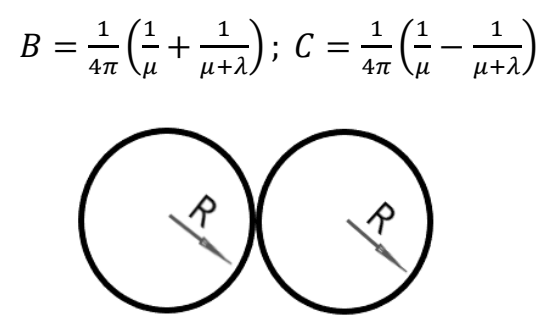

Figure 1: Two sphere in contact

Since the individual particles were part of packing, for the purpose of defining the total stress over the host body, volume concentration (filling ratio of the occupied volume of particles in the cavity) was defined by

$$
\Phi=\frac{4 \pi R^{3} N}{3 V},
$$

where $\mathrm{R}$ is the radius of the individual particle, $\mathrm{N}$ is the number of particles and $\mathrm{V}$ is the volume of the main body.

Then, the initial pressure $(P)$ over the main body was calculated using

$$
P=\frac{\phi C_{N}(-e)^{3 / 2}}{3 \pi^{2} * \frac{1}{4 \pi} *\left(\frac{1}{\mu}+\frac{1}{\mu+\lambda}\right)^{\prime}}
$$

where infinitesimal strain (e) and average coordination number $\left(C_{N}\right)$ were used.

In this study it is proposed to keep hydrostatic pressure constant in the initial state of the tube (which is $\mathrm{P}$ in absence of other static volume forces) for all cases by keeping the unloaded tube physical setup the same. This has been accomplished by setting the cases to have identical initial conditions at the time step when the excitation process starts.

Average coordination number $C_{N}$ was defined as the number of contacts for a particular particle in the cavity. Random packing of same-sized hard spheres are characterised by much smaller packing densities and mean coordination number compared to a regular lattice arrangement ${ }^{10}$. In this study, spherical grains, which were randomly thrown into a box and shaken, took the form of an amorphous arrangement. The packing fraction was found much lower than the densest possible packing level which was mentioned as 'random close packing'11. Since the porosity in the packing was a reason of the coordination number, the regular arrangement of the packing mostly had a certain value; however, there was distribution in the coordination number for the random packing from case to case ${ }^{12}$.

Since the granular structures can easily change their state which means they do not have determined behaviour in the packing, in general, the overall packing is defined as an anisotropic structure. In this study, the individual granules are statistically isotropic structures and they are used to justify the use of isotropic model. This makes possible to consider the packing as an isotropic structure. The Lamế moduli can be introduced for the new isotropic structure with the help of the stress-strain relationship; using the equivalent bulk modulus of this structure as ${ }^{9}$ (Eq. 4).

$$
\lambda^{*}=\frac{\phi N C(-e)^{\frac{1}{2}}}{10 \pi^{2} B(2 B+C)} ; \mu^{*}=\frac{\phi N(5 B+C)(-e)^{1 / 2}}{10 \pi^{2} B(2 B+C)}
$$




\section{Proceedings of the Institute of Acoustics}

$$
k^{*}=\lambda^{*}+\frac{2}{3} \mu^{*}
$$

The equivalent material properties for packed granular structures were smaller than the original material properties of an individual spherical particles. Following the bulk modulus-Young's modulus and -Poisson's ratio relationships, the equivalent material properties were defined in order to model structures in FEM as stated in Equation 6 and Equation 7 for the spherical-rough particles at which equivalent Lamé moduli were substituted into the equation ${ }^{9}$. Equation 8 is the rearranged formula of Equation 7.

$$
\begin{aligned}
& v^{*}=\frac{\lambda^{*}}{2\left(\lambda^{*}+\mu^{*}\right)} \\
& E^{*}=\frac{\mu^{*}\left(3 \lambda^{*}+2 \mu^{*}\right)}{\mu^{*}+\lambda^{*}} \\
& E^{*}=\frac{1-2 v^{*}}{2}\left(\frac{3 E^{2} \phi^{2} C_{N}^{2} p}{\pi^{2}\left(1-v^{* 2}\right)}\right)^{1 / 3}
\end{aligned}
$$

In this study, the spherical particles which had viscoelastic material properties were modelled with the diameter of $15.1 \mathrm{~mm}$ and carbon fibre composite tube $(50 \mathrm{~mm}$ diameter and $300 \mathrm{~mm}$ in height) was used as a container to place the particles. Commercial software EDEM V19.2 based on DEM solver was used as modelling platform.

Filling state of the tube performed in EDEM is shown in Figure 2. Filling process was completed between 0 and $1.5 \mathrm{~s}$. In order to neglect the gravitational effects on the spherical structures and the tube, the excitation was applied from the horizontal direction which was perpendicular to the gravity force. The effect of dynamic load was studied for $1 \mathrm{~s}$ after the filling process is completed which means the excitation was processed from $1.5 \mathrm{~s}$ to $2.5 \mathrm{~s}$. There were totally 68 particles placed in the cavity which constitutes 0.2 filling ratio of the total volume of the tube. Confining pressure and average coordination number were recorded using EDEM postprocessing tools. The material properties (the equivalent Poisson's ratio and the equivalent Young's modulus) had been calculated using these numbers as shown in Eq. 6 and Eq. 8.

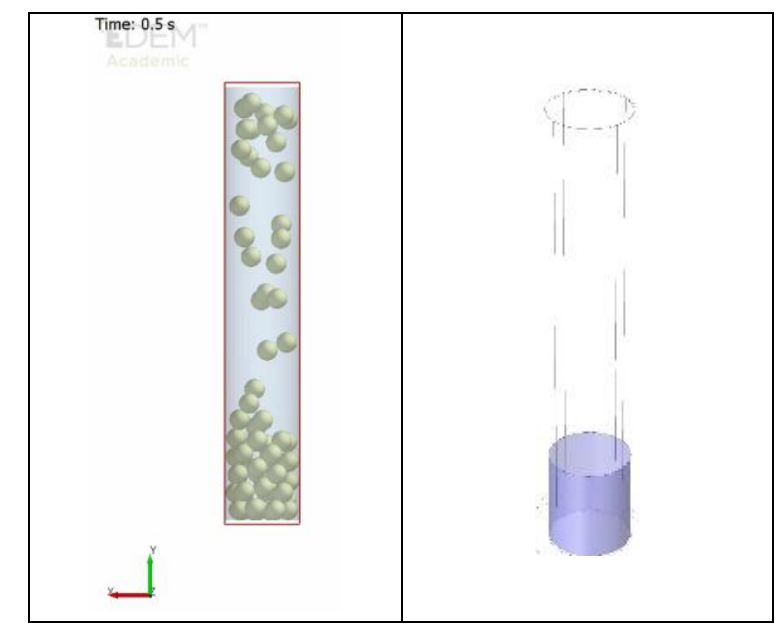

Figure 2: EDEM tube and particle modelling (left), and COMSOL modelling (right)

\subsection{DEM-FEM iteration-based model}

There was an iteration-based approach for the equivalent structure material properties calculation which includes DEM and FEM analysis steps. The first analysis was processed in FEM software in order to find the first bending mode frequency of the empty tube. 
Then, DEM analysis was set with the original material properties at the first bending mode natural frequency of the empty tube. This step was aimed at finding the granular material equivalent property at the given frequency. The equivalent material properties were calculated using Equations 6 and 8.

Then, FEM-based modelling had been used again to fine the updated natural frequency for the cylindrical container partially filled with equivalent material from DEM study. The first bending mode frequency for the new structure was calculated and DEM-based modelling had been recalled updating Young's Modulus at the found natural frequency. The average coordination number and total pressure were taken from EDEM case in order to update Equations 6 and 8 and to find out the equivalent material properties. This DEMFEM based iterative study was followed until the equivalent material properties and the natural frequency converge.

When the granular packing iterative study reached the constant values at the third significant figure for the natural frequency and the material properties, the equivalent material of granular packing can be used as an isotropic solid structure.

In order to show the dependence on displacement amplitude the excitation load was increased from case to case starting at $1^{*} 10^{-5} \mathrm{~m}$ at the first bending mode of the tube partially filled with equivalent material. The loading amplitude was changed through the 13 cases to $25^{\star} 10^{-5} \mathrm{~m}$ in EDEM using the isotropic structure material properties which is given in the last row of Table 1.

Table 1: Iterative case data

\begin{tabular}{|c|c|c|}
\hline Cases & Natural Frequency $[\mathrm{Hz}]$ & Equivalent Young's \\
\hline Original case $\left(1^{\text {st }}\right.$ case $)$ & 733.12 & 1452788.95 \\
\hline $2^{\text {nd }}$ case & 500.41 & 2581389.913 \\
\hline $3^{\text {rd }}$ case & 655.8 & 2582307.354 \\
\hline $4^{\text {th }}$ case & 655.89 & 2582294.366 \\
\hline $5^{\text {th }}$ case & 655.89 & 2582281.811 \\
\hline $6^{\text {th }}$ case & 655.89 & 2582281.811 \\
\hline $7^{\text {th }}$ case & 655.89 & 2582281.811 \\
\hline $8^{\text {th }}$ case & 655.89 & 2582281.811 \\
\hline $9^{\text {th }}$ case & 655.89 & 2582281.811 \\
\hline $10^{\text {th }}$ case & 655.89 & 2582281.811 \\
\hline
\end{tabular}

As it was aforementioned, DEM is a method of studying for the individual bodies in a granular pack which comes with a great computational time and cost. In order to model a continues structure in FEM, which was COMSOL Multiphysics in this study, the least required material properties were density, Young's modulus and Poisson's ratio of the equivalent structure. Figure 2 is used to show the modelled tube with equivalent continuous structure in order to reflect the granular structure placed tube with 0.2 of its volume in FEM. Highlighted volume in Figure 2 shows the equivalent structure in the tube cavity.

\section{RESULTS}

According to the introduced methodology, the equivalent material properties were calculated in iterative study between EDEM and COMSOL Multiphysics. 


\section{Proceedings of the Institute of Acoustics}

These iterations resulted in effective material properties that made possible to replace granular material with equivalent isotropic solid material. The modelled equivalent medium was studied under different amplitude levels using EDEM which is a DEM based software.

According to the iteration based EDEM analysis, the isotropic structure material properties have been reached after a few iterations which can be seen from Table 1. Since the hydrostatic pressure at the initial state of the granular structures was kept at the same level, the material properties depend on the coordination number only. This means the contribution of the particles movement and contact mechanism have great impact on the isotropic structure material property calculations. Figure 3 shows that the coordination number showed stable mechanism after a few EDEM-FEM iterations. Figure 4 supports this information with the stable material properties.

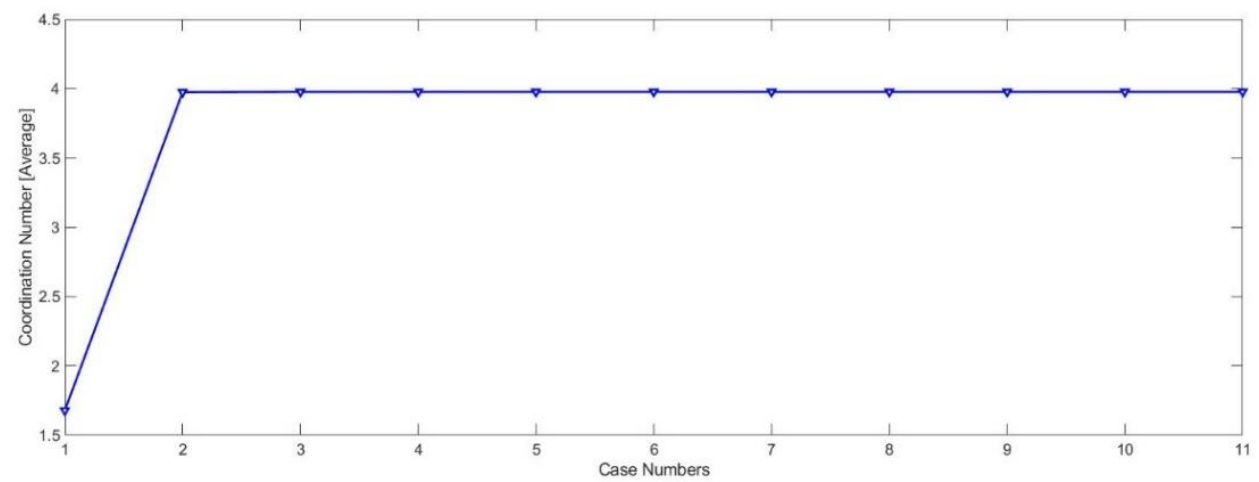

Figure 3: Contact level change in the particles

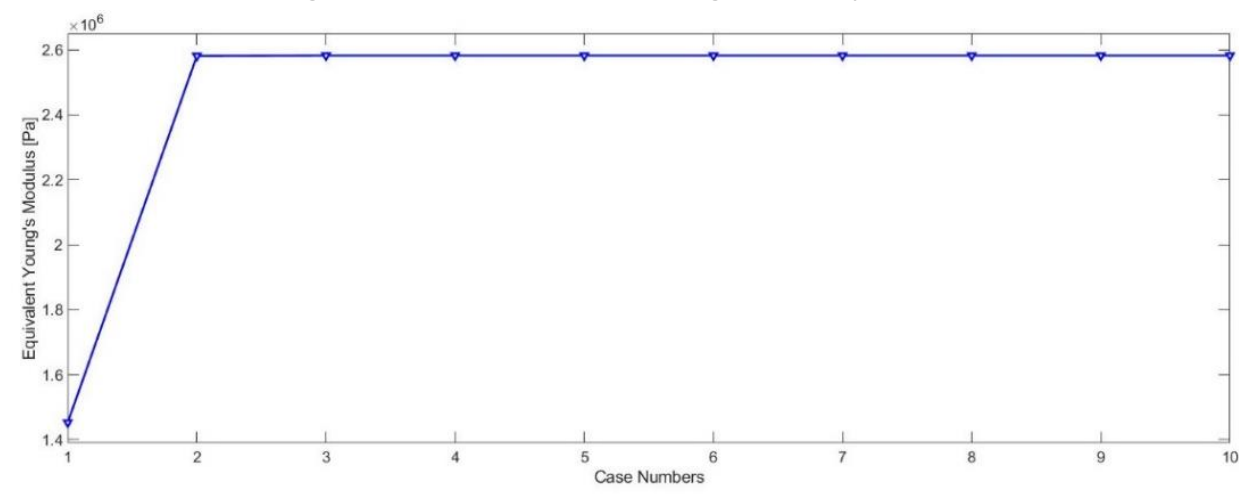

Figure 4: Equivalent Young's Modulus change in iterations

The next result shows that the energy dissipation property was improved using the granular structures in a cavity which was under dynamic excitation. Increased displacement amplitude levels revealed their individual dissipation levels as shown in Figure 5. According to the result, this equivalent material modelling method can be used in a FEM study to predict the loss properties of the solid structure. This study also confirms the amplitude changing behaviour of the particle damper which is known as nonlinearity as it was observed in other literature studies ${ }^{5}$ (Fig 6). 


\section{Proceedings of the Institute of Acoustics}

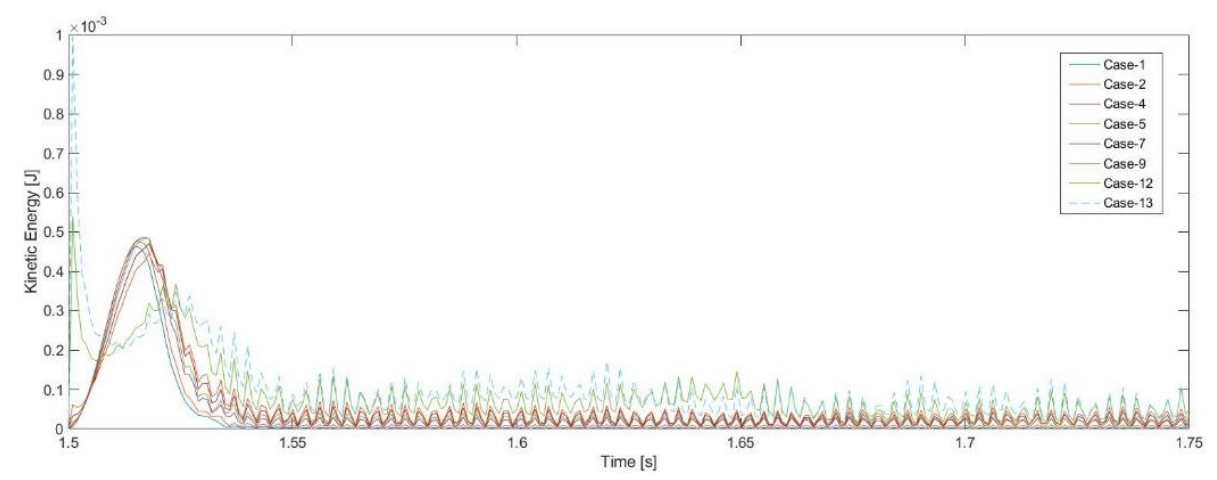

Figure 5: Kinetic energy change rate within amplitude changes

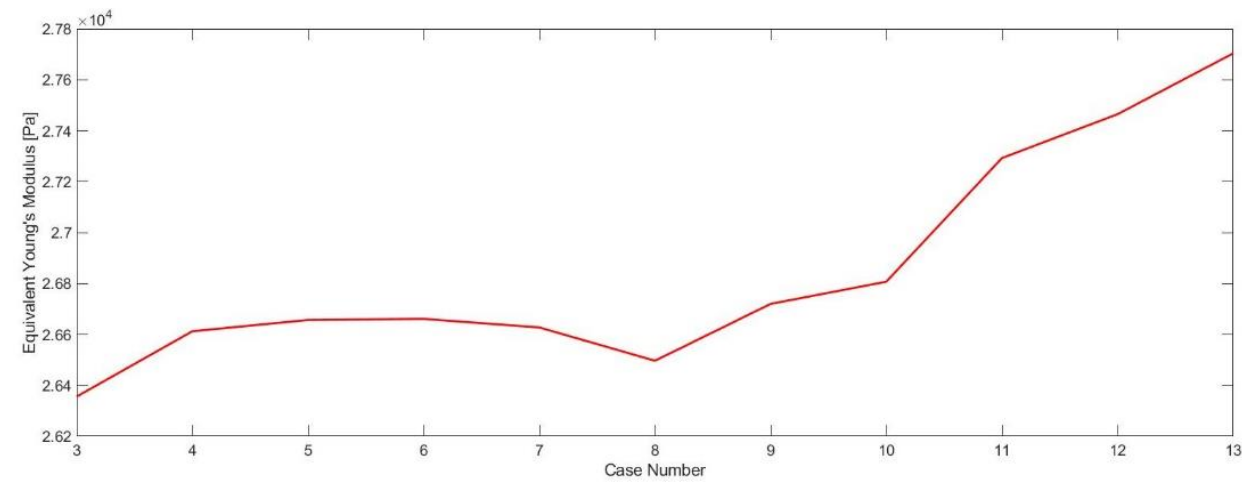

Figure 6: Equivalent material property change within amplitude changes

Kinetic energy dissipation rate of the granular structures is shown in the given result. According to the proposed method, granular structures are modelled in two different ways: DEM and FEM. Using this equivalent material properties defining method, structural vibration studies can be modelled computationally inexpensive and reasonably applicable way in FEM. Further, this study is planned to develop in a study of revealing the loss mechanism and loss factor of the equivalent structure and then arranging them in a periodic arrangement in finite element modelling. By following this study, it is aiming to show the band properties of the offered structure, acoustically. These findings suggest action for the confirmation of experimental and further numerical studies.

\section{REFERENCES}

1. Olson, S. E. An analytical particle damping model. J. Sound Vib. 264, 1155-1166 (2003).

2. Simonian, S. S. Particle beam damper. in Smart Structures and Materials 1995: Passive Damping (ed. Johnson, C. D.) 2445, 149-160 (1995).

3. Lu, Z., Wang, Z., Masri, S. F. \& Lu, X. Particle impact dampers: Past, present, and future. Struct. Control Heal. Monit. 25, 1-25 (2018).

4. Wong, C. \& Rongong, J. Control of particle damper nonlinearity. AIAA J. 47, 953-960 (2009).

5. Rongong, J. A. \& Tomlinson, G. R. Amplitude Dependent Behaviour in the Application of Particle Dampers to Vibrating Structures. 1-9 (2005).

6. Saeki, M. Analytical study of multi-particle damping. J. Sound Vib. 281, 1133-1144

Vol. 42. Pt. 1. 2020 


\section{Proceedings of the Institute of Acoustics}

(2005).

7. Michon, G., Almajid, A. \& Aridon, G. Soft hollow particle damping identification in honeycomb structures. J. Sound Vib. 332, 536-544 (2013).

8. Madan, N., Rojek, J. \& Nosewicz, S. Convergence and stability analysis of the deformable discrete element method. Int. J. Numer. Methods Eng. 118, 320-344 (2019).

9. Walton, K. The Effective Elastic Moduli of A Random Packing of Spheres. J. Mech. Phys. Solids 35, 213-226 (1987).

10. Nolan, G. T. Computer Simulation of Random Packings of Hard Spheres. Powder Technol. 62, 189-196 (1990).

11. Zamponi, F. Packings close and loose. Nature 453, 606-607 (2008).

12. Iwata, H. \& Homma, T. Distribution of coordination numbers in random packing of homogeneous spheres. Powder Technol. 10, 79-83 (1974). 